MATHEMATICS OF COMPUTATION

Volume 67, Number 223, July 1998, Pages 901-916

S $0025-5718(98) 00972-7$

\title{
A NEGATIVE-NORM LEAST SQUARES METHOD FOR REISSNER-MINDLIN PLATES
}

\author{
JAMES H. BRAMBLE AND TONG SUN
}

\begin{abstract}
In this paper a least squares method, using the minus one norm developed by Bramble, Lazarov, and Pasciak, is introduced to approximate the solution of the Reissner-Mindlin plate problem with small parameter $t$, the thickness of the plate. The reformulation of Brezzi and Fortin is employed to prevent locking. Taking advantage of the least squares approach, we use only continuous finite elements for all the unknowns. In particular, we may use continuous linear finite elements. The difficulty of satisfying the inf-sup condition is overcome by the introduction of a stabilization term into the least squares bilinear form, which is very cheap computationally. It is proved that the error of the discrete solution is optimal with respect to regularity and uniform with respect to the parameter $t$. Apart from the simplicity of the elements, the stability theorem gives a natural block diagonal preconditioner of the resulting least squares system. For each diagonal block, one only needs a preconditioner for a second order elliptic problem.
\end{abstract}

\section{INTRODUCTION}

The numerical solution of the Reissner-Mindlin plate model has been discussed by many authors. Many schemes developed early on, using standard finite element methods, are known to possess a locking problem for very thin plates. Using a Helmholtz decomposition, Brezzi and Fortin [11] derived a reformulation of the Reissner-Mindlin plate model, which fundamentally removed the reason for the locking. Another advantage of this reformulation is that the four unknowns are decoupled into two elliptic problems and one mixed saddle point problem. Brezzi and Fortin gave a scheme based on this for which they proved error estimates independent of the plate thickness $t$ for transversal displacement, rotations and shear stresses. The main part of the reformulation is a problem similar to the Stokes problem, so there are some disadvantages when discretizing it directly. First, a discrete saddle point problem has to be solved. Second, the bubble function subspace, $B_{3}$, is used in addition to the linear finite elements, because one cannot independently choose finite element subspaces; the discrete "inf-sup" condition has to be satisfied. Finally, the preconditioning of the resulting system is not simple. Brenner [9] developed a multigrid W-cycle iteration, and proved that when the number of smoothings is large enough, the convergence rate of the iteration does not depend on $t$ or $h$, the mesh size. How many smoothings are required is not clear.

Received by the editor February 7, 1997.

1991 Mathematics Subject Classification. Primary 65N30, 73V05; Secondary 65F10.

Key words and phrases. Plate, locking, least squares, finite element.

(c)1998 American Mathematical Society 
A number of authors tried to develop locking-free schemes which involve only the primitive variables. (Arnold and Falk [1], Duran and Liberman [13], Onate, Zarate and Flores [15], Brezzi, Fortin and Stenberg [12], ...) Each of them used the same framework, a pair of subspaces and a reduction operator [2]. The key point of the analysis is to make the discrete system of the two primitive unknowns equivalent to a discretization of the Brezzi-Fortin reformulation, so that it can be locking free. The choice of the subspace of the rotation is implicitly restricted by the stability condition on the mixed saddle point problem of the Brezzi-Fortin reformulation. The choice of the reduction operator and the subspace of the displacement is restricted by the discrete Helmholtz decomposition, so that the extra unknowns can be eliminated. Therefore the pair of spaces has to be rather complicated. The simplest pair of subspaces is the one given by Onate, Zarate and Flores [15], but Arnold and Falk [2] showed that this method does not converge in the classical sense. Although it is ideal to be able to work on the primitive variables only, as far as computing time is concerned working on the two extra unknowns of the BrezziFortin reformulation does not cost more. In fact, the two elliptic problems can be solved relatively in little time; the solution technique of the Stokes-like problem is also well known.

The other approach is a stabilization method (e.g., [12]). The major measures taken to prevent locking in this approach are the following: (a) A projector which brings the discrete rotation function and the gradient of the displacement function into the same subspace, and (b) the replacement of $t^{-2}$ by $\left(t^{2}+\alpha h^{2}\right)^{-1}$, which is, in a certain sense, equivalent to approximating a thin plate by a thicker plate but keeping the error introduced by this within the magnitude of the discretization error. This approach does produce simpler schemes.

Later, Hughes and Franca [14] and Stenberg [18] developed stabilized mixed formulations which are consistant to the PDE. Therefore they can use balanced elements designed only for interpolation properties. In [18], Stenberg proposed a scheme which is simpler than the one in [14]. The shear stress is removed from both the formulation and the analysis. One possible disadvantage of this approach is that a high regularity assumption on the transversal displacement is needed. But for a plate with partially clamped-partially free boundary conditions, the displacement function may not even be in $H^{2}$ because of the corners of the domain. Besides, the multigrid preconditioners directly based on the formulation of these methods are complicated (cf. [16]), if compared to the diagonal preconditioners.

Arnold, Falk and Winther [4] worked on the preconditioning of these types of schemes. They reported that until now, no attempt to precondition the primitive variable discretizations has been successful. The small parameter $t$ also creates trouble for preconditioning. It appears that even though one can discretize the Reissner-Mindlin plate model with the primitive unknowns only, a locking-free reformulation has to be used for preconditioning, and so additional unknowns have to be used. The paper [4] used a different locking-free reformulation with the shear stress as the new unknown and set up a framework for diagonal preconditioning of the discretization methods described in the paragraphs above. This reformulation also has five scalar unknowns in a saddle point system, but not decoupled. Two of the diagonal blocks of the preconditioner use standard elliptic problem preconditioners. The other one is a preconditioner for $I-t^{2}$ graddiv, which they call $H(d i v)$ preconditioning. The method given in [3] and [4] is restricted to the case in which the pair of subspaces are such that the discrete Helmholtz decomposition holds. 
In this paper, we develop a least squares discretization based on the reformulation of Brezzi and Fortin. A discrete version of $\|\cdot\|_{-1}$ norm [6], [7] is employed for the least squares method to weight the system correctly. The major advantage of least squares methods is that one does not have to satisfy the discrete "infsup" condition and can choose the subspaces for different variables independently. Therefore, continuous elements (e.g., linear finite elements) may be used here for all the unknowns. The error estimates are optimal with respect to regularity and are uniform in $t$. Instead of leading to a saddle point problem, the resulting least squares system is symmetric and positive definite. The stability theorem of the least squares form provides a natural way to precondition the system diagonally. For each block, one only needs to have a preconditioner for a second order elliptic problem, a task which is well understood.

The rest of the paper is organized as follows. Section 2 describes the ReissnerMindlin plate model and the reformulation of Fortin and Brezzi. Section 3 gives the least squares discretization. A stability result on the least squares form is proved in Section 4. The error estimate under full regularity is proved in Section 5. Implementation and numerical calculations are discussed and presented in Sections 6 and 7 .

\section{Reissner-Mindlin PlAte MODEL AND ITS REFORMUlations}

We introduce now some notation for some standard function spaces. Let $L^{2}(\Omega)$ be the square integrable functions on a domain $\Omega \subset \mathbf{R}^{2}$. $\mathbf{L}^{2}(\Omega)=L^{2}(\Omega) \times L^{2}(\Omega)$. The space $\widehat{L}^{2}(\Omega)$ is the space of $L^{2}$ functions with mean value zero; i.e., $p \in \widehat{L}^{2}(\Omega)$ implies that $\int_{\Omega} p=0$. Let $H^{1}(\Omega)$ be the usual Sobolev space of functions whose first derivatives are square integrable. The subspace $H_{0}^{1}(\Omega)$ consists of those functions $u \in H^{1}(\Omega)$ such that $u=0$ on $\partial \Omega$. $\widehat{H}^{1}(\Omega)$ denotes the space of $H^{1}$ functions, $p$, with $\int_{\Omega} p=0$. $\mathbf{H}_{0}^{1}(\Omega)=H_{0}^{1}(\Omega) \times H_{0}^{1}(\Omega)$. $H^{-1}(\Omega)$ is the dual space of $H_{0}^{1}(\Omega)$ with respect of $L^{2}(\Omega)$. $\mathbf{H}^{-1}(\Omega)$ is the dual space of $\mathbf{H}_{0}^{1}(\Omega)$ with respect of $\mathbf{L}^{2}(\Omega)$. The Reissner-Mindlin model of a thin plate with clamped boundary and thickness $t$ is given as the solution of the following problem.

Find $(\omega, \phi) \in H_{0}^{1}(\Omega) \times \mathbf{H}_{0}^{1}(\Omega)$ such that

$$
a(\boldsymbol{\phi}, \boldsymbol{\psi})+\lambda t^{-2}(\boldsymbol{\phi}-\nabla \omega, \boldsymbol{\psi}-\nabla \mu)=(g, \mu), \quad \forall(\mu, \boldsymbol{\psi}) \in H_{0}^{1}(\Omega) \times \mathbf{H}_{0}^{1}(\Omega),
$$

here $(\cdot, \cdot)$ denotes the $\mathbf{L}^{2}$ inner product, and

$$
\begin{aligned}
a(\boldsymbol{\phi}, \psi)=\frac{E}{12\left(1-\nu^{2}\right)} \int_{\Omega}\left[\left(\frac{\partial \phi_{1}}{\partial x}\right.\right. & \left.+\nu \frac{\partial \phi_{2}}{\partial y}\right) \frac{\partial \psi_{1}}{\partial x}+\left(\nu \frac{\partial \phi_{1}}{\partial x}+\frac{\partial \phi_{2}}{\partial y}\right) \frac{\partial \psi_{2}}{\partial y} \\
& \left.+\frac{1-\nu}{2}\left(\frac{\partial \phi_{1}}{\partial y}+\frac{\partial \phi_{2}}{\partial x}\right)\left(\frac{\partial \psi_{1}}{\partial y}+\frac{\partial \psi_{2}}{\partial x}\right)\right] d x d y
\end{aligned}
$$

The constants and variables are:

$\phi$ : rotation of vertical fiber;

$\omega$ : transversal displacement;

$g$ : transversal loading;

$E$ : Young's modulus;

$\nu$ : Poisson's ratio;

$\lambda=\frac{E k}{2(1+\nu)}$ for $0<k<1$. 
By Korn's inequality, there exist constants $C_{1}>C_{0}>0$, such that for any $\phi \in \mathbf{H}_{0}^{1}(\Omega)$,

$$
C_{0} a(\phi, \phi) \leq\left(\nabla \phi_{1}, \nabla \phi_{1}\right)+\left(\nabla \phi_{2}, \nabla \phi_{2}\right) \leq C_{1} a(\phi, \phi) .
$$

Brezzi and Fortin reformulated the Reissner-Mindlin plate problem [11] to avoid the locking phenomenon. By Helmholtz' Theorem, there exist $(r, p) \in H_{0}^{1}(\Omega) \times$ $\widehat{H}^{1}(\Omega)$ such that

$$
\lambda t^{-2}(\nabla \omega-\phi)=\nabla r+\operatorname{curl} p .
$$

Here $\operatorname{curl} p=\left(\frac{\partial p}{\partial y},-\frac{\partial p}{\partial x}\right)$. Using $(2.2)$, one can easily show that $(2.1)$ is equivalent to the following problem.

Find $(r, \phi, p, \omega) \in H_{0}^{1}(\Omega) \times \mathbf{H}_{0}^{1}(\Omega) \times \widehat{H}^{1}(\Omega) \times H_{0}^{1}(\Omega)$, such that

$$
\begin{gathered}
(\nabla r, \nabla \mu)=(g, \mu), \quad \forall \mu \in H_{0}^{1}(\Omega), \\
a(\boldsymbol{\phi}, \boldsymbol{\psi})-(\operatorname{curl} p, \boldsymbol{\psi})=(\nabla r, \boldsymbol{\psi}), \quad \forall \boldsymbol{\psi} \in \mathbf{H}_{0}^{1}(\Omega), \\
-(\boldsymbol{\phi}, \operatorname{curl} q)-t^{2}(\operatorname{curl} p, \operatorname{curl} q)=0, \quad \forall q \in \widehat{H}^{1}(\Omega), \\
(\nabla \omega, \nabla s)=\left(\boldsymbol{\phi}+t^{2} \nabla r, \nabla s\right), \quad \forall s \in H_{0}^{1}(\Omega) .
\end{gathered}
$$

If not otherwise mentioned, we assume that $\Omega$ is a convex polygonal domain. Under this assumption, Arnold and Falk [1] proved the following a priori estimates and regularity results:

$$
\|r\|_{1}+\|\phi\|_{2}+\|p\|_{1}+t\|p\|_{2}+\|\omega\|_{1} \leq C\|g\|_{-1},
$$

and

$$
\|r\|_{2}+\|\omega\|_{2} \leq C\|g\|_{0}, \quad \text { if } g \in L^{2}(\Omega) .
$$

A slight modification of the proof in [1] leads to the following improved estimate for $\omega$ :

$$
\|\omega\|_{2} \leq C\left(\|g\|_{-1}+t^{2}\|g\|_{0}\right) .
$$

It is obvious that (2.3) is an independent Dirichlet problem. Once (2.4) and (2.5) are solved for $\phi$ and $p,(2.6)$ is also easy. The problem is thus reduced to solving the system of (2.4) and (2.5).

For the purpose of approximating the solution of this system we reformulate it as follows. Let $\mathbf{x}^{\perp}=\left(x_{2},-x_{1}\right)$ for $\mathbf{x} \in \mathbf{L}^{2}(\Omega)$. Then

$$
\operatorname{curl} p=\nabla p^{\perp} .
$$

We introduce new variables $\mathbf{f}, \mathbf{u}$ and $\mathbf{v}$ by $\mathbf{f}^{\perp}=\nabla r, \mathbf{u}^{\perp}=\phi$, and $\mathbf{v}^{\perp}=\psi$. Then (2.4) and (2.5) can be rewritten as follows: Find $(p, \mathbf{u}) \in \widehat{H}^{1}(\Omega) \times \mathbf{H}_{0}^{1}(\Omega)$, such that

$$
\begin{array}{ll}
\tilde{a}(\mathbf{u}, \mathbf{v})-(\nabla p, \mathbf{v})=(\mathbf{f}, \mathbf{v}), & \forall \mathbf{v} \in \mathbf{H}_{0}^{1}(\Omega), \\
(\nabla \cdot \mathbf{u}, q)-t^{2}(\nabla p, \nabla q)=0, & \forall q \in \widehat{H}^{1}(\Omega),
\end{array}
$$

where $\tilde{a}(\mathbf{u}, \mathbf{v})=a(\phi, \boldsymbol{\psi})$.

By examining the proof of (2.7) in [1], we have the following estimate:

$$
\|\mathbf{u}\|_{2}+\|p\|_{1}+t\|p\|_{2} \leq C\|\mathbf{f}\| \leq C\left(\|\mathbf{u}\|_{2}+\|p\|_{1}\right),
$$

for $\mathbf{f} \in \mathbf{L}^{2}(\Omega)$. 
Define

$$
A: \mathbf{H}_{0}^{1}(\Omega) \longrightarrow \mathbf{H}^{-1}(\Omega), \quad(A \mathbf{u}, \mathbf{v})=\tilde{a}(\mathbf{u}, \mathbf{v}), \quad \forall \mathbf{v} \in \mathbf{H}_{0}^{1}(\Omega)
$$

and

$$
\Delta: \widehat{H}^{1}(\Omega) \longrightarrow\left(\widehat{H}^{1}(\Omega)\right)^{*}, \quad(\Delta p, q)=-(\nabla p, \nabla q), \quad \forall q \in \widehat{H}^{1}(\Omega) .
$$

The formal version of (2.10) and (2.11) is the following:

$$
\begin{gathered}
A \mathbf{u}-\nabla p=\mathbf{f}, \quad \text { in } \Omega, \\
\nabla \cdot \mathbf{u}+t^{2} \Delta p=0, \quad \text { in } \Omega, \\
\mathbf{u}=0, \quad \frac{\partial p}{\partial n}=0, \quad \text { on } \partial \Omega .
\end{gathered}
$$

\section{A least squares discretization}

In this section we consider a least squares method solving (2.10) and (2.11). Assume that $\Omega$ is quasi-uniformly triangulated. The parameter $h$ is the diameter of the largest of the triangles. Let $V_{h} \subset H_{0}^{1}(\Omega)$ and $\Pi_{h} \subset \widehat{H}^{1}(\Omega)$ be the subspaces consisting of continuous piecewise polynomials of degree $m-1$, with $m \geq 2$, and let $\mathbf{V}_{h}=V_{h} \times V_{h} \in \mathbf{H}_{0}^{1}(\Omega)$. One can choose to use different orders of polynomials for $\mathbf{V}_{h}$ and $\Pi_{h}$. In our numerical computations we use the continuous linear finite elements and hence $m=2$.

First we need to define some discrete operators:

$$
\begin{aligned}
& A_{h}: \mathbf{H}_{0}^{1}(\Omega) \longrightarrow \mathbf{V}_{h}, \quad\left(A_{h} \mathbf{u}, \mathbf{v}\right)=\tilde{a}(\mathbf{u}, \mathbf{v}), \quad \forall \mathbf{v} \in \mathbf{V}_{h} . \\
& \nabla_{h}: \quad L^{2}(\Omega) \longrightarrow \mathbf{V}_{h}, \quad\left(\nabla_{h} p, \mathbf{v}\right)=-(p, \boldsymbol{\nabla} \cdot \mathbf{v}), \quad \forall \mathbf{v} \in \mathbf{V}_{h} \\
& \triangle_{h}: \widehat{H}^{1}(\Omega) \longrightarrow \Pi_{h}, \quad\left(\triangle_{h} p, q\right)=-(\nabla p, \nabla q), \quad \forall q \in \Pi_{h} . \\
& \nabla_{h} . \quad: \quad \mathbf{H}_{0}^{1}(\Omega) \longrightarrow \Pi_{h}, \quad\left(\nabla_{h} \cdot \mathbf{u}, q\right)=-(\mathbf{u}, \nabla q), \quad \forall q \in \Pi_{h} .
\end{aligned}
$$

Define the discrete norm of order minus one, for $\mathbf{v} \in \mathbf{H}^{-1}(\Omega)$, by

$$
\|\mathbf{v}\|_{-1, h}=\sup _{\mathbf{w} \in \mathbf{V}_{h}} \frac{(\mathbf{v}, \mathbf{w})}{\|\mathbf{w}\|_{1}} .
$$

It is easy to prove the following lemma [6].

Lemma 3.1. For any $\mathbf{v} \in \mathbf{V}_{h}$,

$$
\|\mathbf{v}\|_{-1, h}^{2}=\left(T_{h} \mathbf{v}, \mathbf{v}\right),
$$

where $T_{h}: \mathbf{H}^{-1}(\Omega) \longrightarrow \mathbf{V}_{h}$ is defined by $\left(\nabla T_{h} \mathbf{z}, \nabla \mathbf{x}\right)=(\mathbf{z}, \mathbf{x}), \forall \mathbf{x} \in \mathbf{V}_{h}$. Therefore, if $(\cdot, \cdot)_{-1, h}$ is the inner product induced by $\|\cdot\|_{-1, h}$, then

$$
(\mathbf{v}, \mathbf{w})_{-1, h}=\left(T_{h} \mathbf{v}, \mathbf{w}\right), \quad \forall \mathbf{v}, \mathbf{w} \in \mathbf{V}_{h} .
$$

Let $B_{h}: \mathbf{H}^{-1}(\Omega) \longrightarrow \mathbf{V}_{h}$ be an easily computable and spectrally equivalent preconditioner for $T_{h}$. That is,

$$
C_{0}\left(T_{h} \mathbf{v}, \mathbf{v}\right) \leq\left(B_{h} \mathbf{v}, \mathbf{v}\right) \leq C_{1}\left(T_{h} \mathbf{v}, \mathbf{v}\right), \quad \forall \mathbf{v} \in \mathbf{V}_{h},
$$

for some positive constants $C_{0}<C_{1}$ independent of $h$. There is an extensive literature concerning the theory and techniques for building such preconditioners, cf. [5], [10]. 
Our least squares method will be based on the following bilinear form on $\widehat{H}^{1}(\Omega) \times$ $\mathbf{H}_{0}^{1}(\Omega)$ :

$$
\begin{aligned}
\langle\langle(p, \mathbf{u}),(q, \mathbf{v})\rangle\rangle & =\left(B_{h}\left(A_{h} \mathbf{u}-\nabla_{h} p\right), A_{h} \mathbf{v}-\nabla_{h} q\right) \\
& +h^{2}(A \mathbf{u}-\nabla p, A \mathbf{v}-\nabla q)_{h} \\
& +\gamma\left(\nabla_{h} \cdot \mathbf{u}+t^{2} \triangle_{h} p, \nabla_{h} \cdot \mathbf{v}+t^{2} \triangle_{h} q\right)_{M} .
\end{aligned}
$$

Here $\gamma$ is a parameter to be adjusted for balancing and preconditioning the form and $(\cdot, \cdot)_{M}$ is an inner product, equivalent to the $L^{2}$ inner product on $\Pi_{h}$ with constants independent of $h$, chosen to avoid computing the inverse of the mass matrix. The details will be discussed when we describe the implementation. Set $(\mathbf{u}, \mathbf{v})_{h}=\sum_{e \in \Gamma_{h}} \int_{e} \mathbf{u} \cdot \mathbf{v}$, the elementwise inner product. When $\mathbf{u}$ and $\mathbf{v}$ are in $\mathbf{L}^{2}(\Omega),(\mathbf{u}, \mathbf{v})_{h}=(\mathbf{u}, \mathbf{v})$.

The least squares problem is the following: Find $(P, \mathbf{U}) \in \Pi_{h} \times \mathbf{V}_{h}$, such that

$$
\langle\langle(P, \mathbf{U}),(Q, \mathbf{V})\rangle\rangle=\left(B_{h} \mathbf{f}, A_{h} \mathbf{V}-\nabla_{h} Q\right)+h^{2}(\mathbf{f}, A \mathbf{V}-\nabla Q)_{h},
$$

for all $(Q, \mathbf{V}) \in \Pi_{h} \times \mathbf{V}_{h}$.

\section{Stability}

First we prove a stability result on the least squares bilinear form (3.8). For the rest of the paper, $C$ will stand for a constant independent of both $h$ or $t$ which may take on different values at different occurrences.

Theorem 4.1. For any $(P, \mathbf{U}) \in \Pi_{h} \times \mathbf{V}_{h}$,

$$
\begin{aligned}
\|\mathbf{U}\|_{1} & +\|P\|+t\|P\|_{1}+t^{2}\left\|\triangle_{h} P\right\| \\
& \leq C\left\{\left\|A_{h} \mathbf{U}-\nabla_{h} P\right\|_{-1, h}+h\|A \mathbf{U}-\nabla P\|_{h}+\left\|\nabla_{h} \cdot \mathbf{U}+t^{2} \triangle_{h} P\right\|\right\} \\
& \leq C\langle\langle(P, \mathbf{U}),(P, \mathbf{U})\rangle\rangle^{\frac{1}{2}} \\
& \leq C\left(\|\mathbf{U}\|_{1}+\|P\|+t\|P\|_{1}+t^{2}\left\|\triangle_{h} P\right\|\right),
\end{aligned}
$$

where $\|\cdot\|_{h}=(\cdot, \cdot)_{h}^{\frac{1}{2}}$.

Proof. We prove the first inequality; the second and third are obvious. It is well known that for all $p \in \widehat{L}^{2}(\Omega)$,

$$
\|p\| \leq \sup _{\mathbf{v} \in \mathbf{H}_{0}^{1}(\Omega)} \frac{(p, \nabla \cdot \mathbf{v})}{\|\mathbf{v}\|_{1}} .
$$

This result may be found in Girault and Raviart [17]. Fix $P \in \Pi_{h}, \mathbf{v} \in \mathbf{H}_{0}^{1}(\Omega)$. Let $\mathbf{w} \in \mathbf{V}_{h}$ satisfy

$$
h\|\mathbf{w}\|_{1}+\|\mathbf{v}-\mathbf{w}\| \leq C h\|\mathbf{v}\|_{1} .
$$

Then

$$
\begin{aligned}
|(P, \nabla \cdot \mathbf{v})| & =|(P, \nabla \cdot(\mathbf{v}-\mathbf{w}))+(P, \nabla \cdot \mathbf{w})| \\
& \leq|(\nabla P, \mathbf{v}-\mathbf{w})|+\left|\left(\nabla_{h} P, \mathbf{w}\right)\right|
\end{aligned}
$$

and

$$
\begin{gathered}
|(\nabla P, \mathbf{v}-\mathbf{w})| \leq\|\nabla P\|\|\mathbf{v}-\mathbf{w}\| \leq C h\|\nabla P\|_{h}\|\mathbf{v}\|_{1}, \\
\left|\left(\nabla_{h} P, \mathbf{w}\right)\right| \leq\left\|\nabla_{h} P\right\|_{-1, h}\|\mathbf{w}\|_{1} \leq C\left\|\nabla_{h} P\right\|_{-1, h}\|\mathbf{v}\|_{1} .
\end{gathered}
$$


Hence

$$
\begin{aligned}
\|P\| & \leq C\left(\left\|\nabla_{h} P\right\|_{-1, h}+h\|\nabla P\|_{h}\right) . \\
& \leq C\left(\left\|A_{h} \mathbf{U}-\nabla_{h} P\right\|_{-1, h}+h\|A \mathbf{U}-\nabla P\|_{h}+\|\mathbf{U}\|_{1}\right) .
\end{aligned}
$$

Here we have used the inverse property $h\|\mathbf{U}\|_{k} \leq C\|\mathbf{U}\|_{k-1}$ for $\mathbf{U} \in \mathbf{V}_{h}$.

For $\mathbf{U} \in \mathbf{V}_{h}$, according to Korn's inequality,

$$
\begin{aligned}
C\|\mathbf{U}\|_{1}^{2} & \leq \tilde{a}(\mathbf{U}, \mathbf{U})=\left(A_{h} \mathbf{U}, \mathbf{U}\right) \\
& =\left(A_{h} \mathbf{U}-\nabla_{h} P, \mathbf{U}\right)+\left(\nabla_{h} P, \mathbf{U}\right) \\
& =\left(A_{h} \mathbf{U}-\nabla_{h} P, \mathbf{U}\right)-\left(P, \nabla_{h} \cdot \mathbf{U}\right) \\
& =\left(A_{h} \mathbf{U}-\nabla_{h} P, \mathbf{U}\right)-\left(P, \nabla_{h} \cdot \mathbf{U}+t^{2} \triangle_{h} P\right)+t^{2}\left(P, \triangle_{h} P\right) .
\end{aligned}
$$

Thus

$$
\begin{aligned}
& C\|\mathbf{U}\|_{1}^{2}+t^{2}\|P\|_{1}^{2} \\
& \quad \leq\left\|A_{h} \mathbf{U}-\nabla_{h} P\right\|_{-1, h}\|\mathbf{U}\|_{1}+\|P\|\left\|\nabla_{h} \cdot \mathbf{U}+t^{2} \triangle_{h} P\right\| .
\end{aligned}
$$

Combining the last inequality and the estimate for $\|P\|$ above, using the Schwarz inequality and some simple manipulations, we get

$$
\begin{aligned}
& \|\mathbf{U}\|_{1}+\|P\|+t\|P\|_{1} \\
& \quad \leq C\left\{\left\|A_{h} \mathbf{U}-\nabla_{h} P\right\|_{-1, h}+h\|A \mathbf{U}-\nabla P\|_{h}+\left\|\nabla_{h} \cdot \mathbf{U}+t^{2} \triangle_{h} P\right\|\right\} .
\end{aligned}
$$

Now it is easy to see that the first inequality of the theorem holds and the theorem is proved.

This first theorem implies that the least squares form (3.8) is positive definite and hence the least squares problem (3.9) has a unique solution.

\section{ERror estimates}

Theorem 5.1. Let $(p, \mathbf{u})$ be the solution of $(2.10)$ and $(2.11)$, and $(P, \mathbf{U})$ be the solution of (3.9). Assume that $\mathbf{f} \in \mathbf{L}^{2}(\Omega),(p, \mathbf{u}) \in\left(H^{m}(\Omega) \cap \widehat{H}^{1}(\Omega)\right) \times$ $\left(\mathbf{H}^{m}(\Omega) \cap \mathbf{H}_{0}^{1}(\Omega)\right)$, and that $\mathbf{V}_{h}$ and $\Pi_{h}$ consist of continuous piecewise polynomials of degree $m-1, m \geq 2$. Then

$$
\|\mathbf{u}-\mathbf{U}\|_{1}+\|p-P\|+t\|p-P\|_{1} \leq C h^{m-1}\left(\|\mathbf{u}\|_{m}+\|p\|_{m-1}+t\|p\|_{m}\right) .
$$

In particular, for $m=2$,

$$
\|\mathbf{u}-\mathbf{U}\|_{1}+\|p-P\|+t\|p-P\|_{1} \leq C h\|\mathbf{f}\| .
$$

Proof. Now

$$
\tilde{a}(\mathbf{u}, \mathbf{v})-(\nabla p, \mathbf{v})=(\mathbf{f}, \mathbf{v}), \quad \forall \mathbf{v} \in \mathbf{H}_{0}^{1}(\Omega)
$$

so that

$$
\left(A_{h} \mathbf{u}-\nabla_{h} p, \mathbf{V}\right)=(\mathbf{f}, \mathbf{V}), \quad \forall \mathbf{V} \in \mathbf{V}_{h} .
$$

By (5.1) and density,

$$
(A \mathbf{u}-\nabla p, \mathbf{v})=(\mathbf{f}, \mathbf{v}), \quad \forall \mathbf{v} \in \mathbf{L}^{2}(\Omega) .
$$


Moreover, for any $Q \in \Pi_{h} \subset \widehat{H}^{1}(\Omega)$,

$$
\left(\nabla_{h} \cdot \mathbf{u}+t^{2} \triangle_{h} p, Q\right)=-(\mathbf{u}, \nabla Q)-t^{2}(\nabla p, \nabla Q)=(\nabla \cdot \mathbf{u}, Q)-t^{2}(\nabla p, \nabla Q)=0,
$$

and therefore

$$
\nabla_{h} \cdot \mathbf{u}+t^{2} \triangle_{h} p=0 .
$$

It follows from (3.9), (5.2), (5.3) and (5.4) that

$$
\langle\langle(e, \mathbf{E}),(Q, \mathbf{V})\rangle\rangle=0, \quad \forall(Q, \mathbf{V}) \in \Pi_{h} \times \mathbf{V}_{h},
$$

where $e=p-P$ and $\mathbf{E}=\mathbf{u}-\mathbf{U}$. Define $\tilde{P} \in \Pi_{h}$ by

$$
(\nabla \tilde{P}, \nabla Q)=(\nabla p, \nabla Q), \quad \forall Q \in \Pi_{h} .
$$

Now $\tilde{P}$ is the $\widehat{H}^{1}(\Omega)$ projection of $p$ into $\Pi_{h}$. Thus, since for some $\bar{P} \in \Pi_{h}$,

$$
\|p-\bar{P}\|_{1} \leq C h^{l-1}\|p\|_{l}, \quad l \leq m
$$

we have

$$
\|p-\tilde{P}\|_{1} \leq\|p-\bar{P}\|_{1} \leq C h^{l-1}\|p\|_{l}, \quad l \leq m .
$$

Moreover, by the standard duality argument (Aubin-Nitsche trick), we have

$$
\|p-\tilde{P}\| \leq C h^{m-1}\|p\|_{m-1} .
$$

Choose $\tilde{\mathbf{U}} \in \mathbf{V}_{h}$ such that

$$
\|\mathbf{u}-\tilde{\mathbf{U}}\|+h\|\mathbf{u}-\tilde{\mathbf{U}}\|_{1}<C h^{m}\|\mathbf{u}\|_{m}
$$

and let

$$
(\tilde{e}, \tilde{\mathbf{E}})=(\tilde{P}-P, \tilde{\mathbf{U}}-\mathbf{U}) .
$$

By the stability theorem and (5.5) we have that,

$$
\begin{aligned}
\|\tilde{\mathbf{E}}\|_{1}+\|\tilde{e}\|+t\|\tilde{e}\|_{1} & \leq C\langle\langle(\tilde{e}, \tilde{\mathbf{E}}),(\tilde{e}, \tilde{\mathbf{E}})\rangle\rangle^{1 / 2} \\
& =C\langle\langle(\tilde{P}-p, \tilde{\mathbf{U}}-\mathbf{u}),(\tilde{e}, \tilde{\mathbf{E}})\rangle\rangle^{1 / 2} .
\end{aligned}
$$

Hence,

$$
\begin{aligned}
\|\tilde{\mathbf{E}}\|_{1}+\|\tilde{e}\|+t\|\tilde{e}\|_{1} \leq & C\langle\langle(\tilde{e}, \tilde{\mathbf{E}}),(\tilde{e}, \tilde{\mathbf{E}})\rangle\rangle^{1 / 2} \\
\leq & C\langle\langle(\tilde{P}-p, \tilde{\mathbf{U}}-\mathbf{u}),(\tilde{P}-p, \tilde{\mathbf{U}}-\mathbf{u})\rangle\rangle^{1 / 2} \\
\leq & C\left\{\left\|A_{h}(\tilde{\mathbf{U}}-\mathbf{u})-\nabla_{h}(\tilde{P}-p)\right\|_{-1, h}\right. \\
& +h\|A(\tilde{\mathbf{U}}-\mathbf{u})-\nabla(\tilde{P}-p)\|_{h} \\
& \left.+\left\|\nabla_{h} \cdot(\tilde{\mathbf{U}}-\mathbf{u})+t^{2} \triangle_{h}(\tilde{P}-p)\right\|\right\} .
\end{aligned}
$$

But

$$
\begin{gathered}
\left\|A_{h}(\tilde{\mathbf{U}}-\mathbf{u})\right\|_{-1, h} \leq C\|\tilde{\mathbf{U}}-\mathbf{u}\|_{1} \leq C h^{m-1}\|\mathbf{u}\|_{m}, \\
\left\|\boldsymbol{\nabla}_{h}(\tilde{P}-p)\right\|_{-1, h} \leq\|\tilde{P}-p\| \leq C h^{m-1}\|p\|_{m-1}, \\
h\|\boldsymbol{\nabla}(\tilde{P}-p)\|_{h} \leq h\|\tilde{P}-p\|_{1} \leq C h^{m-1}\|p\|_{m-1} .
\end{gathered}
$$

In [7], it is shown that

$$
h\|A(\tilde{\mathbf{U}}-\mathbf{u})\|_{h} \leq C h^{m-1}\|\mathbf{u}\|_{m} .
$$

For $m=2$, the inequality above is trivial. Because of the definition of $\tilde{P}$,

$$
\triangle_{h} \tilde{P}=\triangle_{h} p,
$$


and thus we have

$$
\begin{aligned}
& \left\|\nabla_{h} \cdot(\tilde{\mathbf{U}}-\mathbf{u})+t^{2} \triangle_{h}(\tilde{P}-p)\right\| \\
& \quad=\left\|\nabla_{h} \cdot(\tilde{\mathbf{U}}-\mathbf{u})\right\| \leq C\|\tilde{\mathbf{U}}-\mathbf{u}\|_{1} \leq C h^{m-1}\|\mathbf{u}\|_{m} .
\end{aligned}
$$

Therefore,

$$
\|\tilde{\mathbf{E}}\|_{1}+\|\tilde{e}\|+t\|\tilde{e}\|_{1} \leq C h^{m-1}\left(\|\mathbf{u}\|_{m}+\|p\|_{m-1}\right)
$$

By (5.10), (5.8), (5.9), (5.11), and the triangle inequality,

$$
\|\mathbf{E}\|_{1}+\|e\|+t\|e\|_{1} \leq C h^{m-1}\left(\|\mathbf{u}\|_{m}+\|p\|_{m-1}+t\|p\|_{m}\right) .
$$

This proves the theorem.

Next we give an error estimate theorem for the solution of the complete system.

Theorem 5.2. Let $(r, \boldsymbol{\phi}, p, \omega) \in H_{0}^{1}(\Omega) \times \mathbf{H}_{0}^{1}(\Omega) \times \widehat{H}^{1}(\Omega) \times H_{0}^{1}(\Omega)$ be the solution of system (2.3), (2.4), (2.5) and (2.6). $\left(r_{h}, \phi_{h}, p_{h}, \omega_{h}\right) \in V_{h} \times \mathbf{V}_{h} \times \Pi_{h} \times V_{h}, \mathbf{u}_{h}^{\perp}=\phi_{h}$, and $\mathbf{f}_{h}^{\perp}=\nabla r_{h}$, such that

$$
\begin{gathered}
\left(\nabla r_{h}, \nabla \mu\right)=(g, \mu), \\
\left\langle\left\langle\left(p_{h}, \mathbf{u}_{h}\right),(Q, \mathbf{V})\right\rangle\right\rangle=\left(B_{h} \mathbf{f}_{h}, A_{h} \mathbf{V}-\nabla_{h} Q\right)+h^{2}\left(\mathbf{f}_{h}, A \mathbf{V}-\nabla Q\right)_{h},
\end{gathered}
$$

for all $\mu \in V_{h},(Q, \mathbf{V}) \in \Pi_{h} \times \mathbf{V}_{h}$ and $s \in V_{h}$. Then

$$
\begin{gathered}
\left\|r-r_{h}\right\|_{1} \leq C h\|g\|, \\
\left\|r-r_{h}\right\|+\left\|\mathbf{u}-\mathbf{u}_{h}\right\|_{1}+\left\|p-p_{h}\right\|+t\left\|p-p_{h}\right\|_{1} \leq C h\|g\|_{-1}, \\
\left\|\omega-\omega_{h}\right\|_{1} \leq C h\left(\|g\|_{-1}+t^{2}\|g\|\right) .
\end{gathered}
$$

Proof. The first estimate for $r-r_{h}$ is standard. By the duality argument we also have

$$
\left\|r-r_{h}\right\| \leq C h\|g\|_{-1} .
$$

Let $(\tilde{\mathbf{u}}, \tilde{p})$ be the solution of (2.10) and (2.11) with $\mathbf{f}$ replaced by $\mathbf{f}_{h}$. Then,

$$
\begin{gathered}
\tilde{a}(\mathbf{u}-\tilde{\mathbf{u}}, \mathbf{v})-(\nabla(p-\tilde{p}), \mathbf{v})=\left(\mathbf{f}-\mathbf{f}_{h}, \mathbf{v}\right), \quad \forall \mathbf{v} \in \mathbf{H}_{0}^{1}(\Omega), \\
(\nabla \cdot(\mathbf{u}-\tilde{\mathbf{u}}), q)-t^{2}(\nabla(p-\tilde{p}), \nabla q)=0, \quad \forall q \in \widehat{H}^{1}(\Omega) .
\end{gathered}
$$

Let $\mathbf{v}=\mathbf{u}-\tilde{\mathbf{u}}$ and $q=p-\tilde{p}$ in the two equations. Adding them together we have,

$$
\tilde{a}(\mathbf{u}-\tilde{\mathbf{u}}, \mathbf{u}-\tilde{\mathbf{u}})+t^{2}(\nabla(p-\tilde{p}), \nabla(p-\tilde{p}))=\left(\mathbf{f}-\mathbf{f}_{h}, \mathbf{u}-\tilde{\mathbf{u}}\right),
$$

from which it follows that,

$$
\|\mathbf{u}-\tilde{\mathbf{u}}\|_{1}+t\|p-\tilde{p}\|_{1} \leq C\left\|\mathbf{f}-\mathbf{f}_{h}\right\|_{-1} .
$$

In order to bound the $L^{2}$ norm of $p-\tilde{p}$ we use (4.1). Thus

$$
\begin{aligned}
\|p-\tilde{p}\| & \leq C \sup _{\mathbf{v} \in \mathbf{H}_{0}^{1}(\Omega)} \frac{(\nabla(p-\tilde{p}), \mathbf{v})}{\|\mathbf{v}\|_{1}} \\
& =C \sup _{\mathbf{v} \in \mathbf{H}_{0}^{1}(\Omega)} \frac{\left(\mathbf{f}-\mathbf{f}_{h}, \mathbf{v}\right)-\tilde{a}(\mathbf{u}-\tilde{\mathbf{u}}, \mathbf{v})}{\|\mathbf{v}\|_{1}} \\
& \leq C\left(\left\|\mathbf{f}-\mathbf{f}_{h}\right\|_{-1}+\|\mathbf{u}-\tilde{\mathbf{u}}\|_{1}\right) .
\end{aligned}
$$


But

$$
\left\|\mathbf{f}-\mathbf{f}_{h}\right\|_{-1}=\left\|\nabla\left(r-r_{h}\right)\right\|_{-1} \leq\left\|r-r_{h}\right\| \leq C h\|g\|_{-1} .
$$

Hence

$$
\|\mathbf{u}-\tilde{\mathbf{u}}\|_{1}+\|p-\tilde{p}\|+t\|p-\tilde{p}\|_{1} \leq C\left\|\mathbf{f}-\mathbf{f}_{h}\right\|_{-1} \leq C h\|g\|_{-1} .
$$

By the previous theorem,

$$
\left\|\mathbf{u}_{h}-\tilde{\mathbf{u}}\right\|_{1}+\left\|p_{h}-\tilde{p}\right\|+t\left\|p_{h}-\tilde{p}\right\|_{1} \leq C h\left\|\mathbf{f}_{h}\right\| \leq C h\|g\|_{-1} .
$$

Now (5.18) and (5.19) imply that

$$
\left\|\mathbf{u}_{h}-\mathbf{u}\right\|_{1}+\left\|p_{h}-p\right\|+t\left\|p_{h}-p\right\|_{1} \leq C h\|g\|_{-1},
$$

which completes the proof of the next to last inequality of the theorem.

Finally, we need to prove the estimate for $\omega-\omega_{h}$. Let $\tilde{\omega}$ be the solution of

$$
(\nabla \tilde{\omega}, \nabla s)=\left(\phi_{h}+t^{2} \nabla r, \nabla s\right), \quad \forall s \in H_{0}^{1}(\Omega) .
$$

Then $\tilde{\omega} \in H^{2}(\Omega)$ and

$$
\|\omega-\tilde{\omega}\|_{1} \leq\left\|\phi-\phi_{h}\right\| \leq C h\|g\|_{-1} .
$$

Let $\theta=\tilde{\omega}-t^{2} r, \theta_{h}=\omega_{h}-t^{2} r_{h}$, then $\theta$ and $\theta_{h}$ are solutions of the problems

$$
(\nabla \theta, \nabla s)=\left(\phi_{h}, \nabla s\right), \quad \forall s \in H_{0}^{1}(\Omega),
$$

and

$$
\left(\nabla \theta_{h}, \nabla s\right)=\left(\phi_{h}, \nabla s\right), \quad \forall s \in V_{h},
$$

respectively. (Here we used the fact that both $r_{h}$ and $\omega_{h}$ are in the same subspace $V_{h}$.) It is standard that

$$
\left\|\theta-\theta_{h}\right\|_{1} \leq C h\left\|\nabla \cdot \phi_{h}\right\| \leq C h\|g\|_{-1},
$$

and

$$
\left\|\tilde{\omega}-\omega_{h}\right\|_{1} \leq\left\|\theta-\theta_{h}\right\|_{1}+t^{2}\left\|r-r_{h}\right\|_{1} \leq C h\left(\|g\|_{-1}+t^{2}\|g\|\right) .
$$

The proof is completed by combining (5.22) and (5.23).

If we replace $\mathbf{f}_{h}^{\perp}=\nabla r_{h}$ by $\mathbf{f}_{h}^{\perp}=\nabla_{h} r_{h}$ in this theorem, the theorem holds. In fact, the only thing that needs to be verified is (5.17) and (5.19). We observe that

$$
\begin{aligned}
\left\|\mathbf{f}-\mathbf{f}_{h}\right\|_{-1} & =\left\|\nabla r-\nabla_{h} r_{h}\right\|_{-1} \\
& \leq C \sup _{\mathbf{v} \in \mathbf{H}_{0}^{1}(\Omega)} \frac{\left(\nabla r-\nabla_{h} r_{h}, \mathbf{v}\right)}{\|\mathbf{v}\|_{1}} \\
& =C \sup _{\mathbf{v} \in \mathbf{H}_{0}^{1}(\Omega)} \frac{\left(\nabla r-\nabla_{h} r_{h}, \mathbf{v}-\mathbf{v}_{h}\right)+\left(\nabla r-\nabla_{h} r_{h}, \mathbf{v}_{h}\right)}{\|\mathbf{v}\|_{1}} \\
& \leq C\left(\|r\|_{1} h+\left\|r-r_{h}\right\|\right) \\
& \leq C h\|g\|_{-1} .
\end{aligned}
$$

Here $\mathbf{v}_{h} \in \mathbf{V}_{h}$ is chosen to satisfy

$$
h\left\|\mathbf{v}_{h}\right\|_{1}+\left\|\mathbf{v}-\mathbf{v}_{h}\right\| \leq C h\|\mathbf{v}\|_{1} .
$$

It is obvious that $\left\|\nabla_{h} r_{h}\right\| \leq\left\|\nabla r_{h}\right\| \leq\|\nabla r\|$.

Although this replacement seems unnecessary and computationally uneconomical, it might be needed to prove an error estimate under weaker regularity assumptions. 


\section{IMPLEMENTATION}

First we set up some terminology. A partial differential equation can be described as

$$
L w=z,
$$

where $w \in H_{E}$ is the solution, $H_{E}$ is a Sobolev space, $z \in F$ is the right hand side, $F \subset H_{E}^{*}, H_{E}^{*}$ is the dual space of $H_{E}$ with respect to $L^{2}$ and $L: H_{E} \rightarrow F$ is the differential operator.

A conforming finite element subspace $W_{h}$ is a finite dimensional subspace of $H_{E}$. Let $\left\{\varphi^{1}, \cdots, \varphi^{m}\right\}$ be a basis of $W_{h}$. If a function $w \in W_{h}$ is an approximate solution, a correction to be added to an approximate solution, or a test function in a weak formulation, we usually compute its coefficient vector $w_{c}=\left(\alpha_{j}\right)$ under the basis $\left\{\varphi^{1}, \cdots, \varphi^{m}\right\}$, defined by $w=\sum_{j=1}^{m} \alpha_{j} \varphi^{j}$. For a function $z$ in $W_{h}$ or $F$, which is the right hand side, a residual, or an image of $L$, we usually need to compute its dual vector $z_{d}=\left(\beta^{i}\right)$, with $\beta^{i}=\left(\varphi^{i}, z\right)$. In the weak formulation

$$
(L w, v)=(z, v), \quad \forall v \in H_{E},
$$

where $L w$ and $z$ are in the dual space of $H_{E}$. So, it is only natural to consider their dual vectors. Although the $L^{2}$ projections of $L w$ and $z$ into $W_{h}$ are in $W_{h}$, they still represent functionals on $W_{h}$, not functions in $W_{h}$. And it still does not make sense to consider their coefficient vectors under a basis.

It is well known that, as a matrix action, a preconditioner takes a dual vector and produces a coefficient vector.

1. The computation of the bilinear form

$$
\left(B_{h}\left(A_{h} \mathbf{U}-\nabla_{h} P\right), A_{h} \mathbf{V}-\nabla_{h} Q\right)
$$

has been discussed in [7]. For the convenience of the readers, we include a discussion about it here. Let $\left\{\xi^{1}, \cdots, \xi^{n}\right\}$ be a basis of $\mathbf{V}_{h}$, and $\left\{\eta^{1}, \cdots, \eta^{k}\right\}$ be a basis of $\Pi_{h} \oplus \mathbf{R}$. For $\mathbf{U}, \mathbf{V} \in \mathbf{V}_{h}$ and $P, Q \in \Pi_{h} \oplus \mathbf{R}, \mathbf{U}_{c}, \mathbf{V}_{c}$ and $P_{c}, Q_{c}$ are the coefficient vectors of $\mathbf{U}, \mathbf{V}$ and $P, Q$, respectively. To compute $\left(B_{h}\left(A_{h} \mathbf{U}-\nabla_{h} P\right), A_{h} \mathbf{V}-\nabla_{h} Q\right)$, first we need to compute the entries of the the dual vector of $A_{h} \mathbf{U}-\nabla_{h} P$ :

$$
\left(\xi^{i}, A_{h} \mathbf{U}-\nabla_{h} P\right)=\widetilde{a}\left(\xi^{i}, \mathbf{U}\right)+\left(\nabla \cdot \xi^{i}, P\right)=\left(K \mathbf{U}_{c}\right)_{i}+\left(S P_{c}\right)_{i},
$$

where $K=\left(\widetilde{a}\left(\xi^{i}, \boldsymbol{\xi}^{j}\right)\right)$ is the stiffness matrix, $S=\left(\left(\nabla \cdot \xi^{i}, \boldsymbol{\eta}^{j}\right)\right)$.

Let $\mathbf{w}_{c}$ be the coefficient vector of $\mathbf{w}=B_{h}\left(A_{h} \mathbf{U}-\nabla_{h} P\right)$, then $\mathbf{w}_{c}=$ $B_{1}\left(K \mathbf{U}_{c}+S P_{c}\right)$, where $B_{1}$ is the matrix corresponding to $B_{h}$. Now it is easy to see that

$$
\begin{aligned}
\left(B_{h}(\right. & \left.\left.A_{h} \mathbf{U}-\nabla_{h} P\right), A_{h} \mathbf{V}-\nabla_{h} Q\right) \\
& =\widetilde{a}(\mathbf{w}, \mathbf{V})+(\nabla \cdot \mathbf{w}, Q) \\
& =\widetilde{a}(\mathbf{V}, \mathbf{w})+(Q, \nabla \cdot \mathbf{w}) \\
& =\mathbf{V}_{c}^{T} K \mathbf{w}_{c}+Q_{c}^{T} S^{T} \mathbf{w}_{c} \\
& =\left(\mathbf{V}_{c}^{T} K+Q_{c}^{T} S^{T}\right) B_{1}\left(K \mathbf{U}_{c}+S P_{c}\right) \\
& =\left(\begin{array}{c}
\mathbf{V}_{c} \\
Q_{c}
\end{array}\right)^{T}\left(\begin{array}{c}
K \\
S^{T}
\end{array}\right) B_{1}\left(\begin{array}{ll}
K & S
\end{array}\right)\left(\begin{array}{c}
\mathbf{U}_{c} \\
P_{c}
\end{array}\right) .
\end{aligned}
$$


The matrix $\mathcal{A}_{1}=\left(\begin{array}{c}K \\ S^{T}\end{array}\right) B_{1}\left(\begin{array}{ll}K & S\end{array}\right)$ is usually a full matrix, which is not easily assembled. But its action on a coefficient vector of the form $\left(\begin{array}{c}\mathbf{U}_{c} \\ P_{c}\end{array}\right)$ is clearly computable.

2. Now we consider the computation of

$$
\left(\nabla_{h} \cdot \mathbf{U}+t^{2} \triangle_{h} P, \nabla_{h} \cdot \mathbf{V}+t^{2} \triangle_{h} Q\right)_{M} .
$$

The inner product $(\cdot, \cdot)_{M}$ is defined as follows.

Suppose that $R \in \Pi_{h}$ is given in the form of $\nabla_{h} \cdot \mathbf{U}$ or $\triangle_{h} P$. Then it is only convenient to compute its dual vector. To get the coefficient vector, one has to multiply the dual vector by the inverse of the mass matrix $M=\left(\left(\eta^{i}, \eta^{j}\right)\right)$.

Let $\mathbf{U}_{c}, \mathbf{V}_{c}$ and $P_{c}, Q_{c}$ be the coefficient vectors of $\mathbf{U}, \mathbf{V} \in \mathbf{V}_{h}$ and $P, Q \in \Pi_{h}$, respectively, as above. It is clear that the dual vector of $\nabla_{h} \cdot \mathbf{U}+t^{2} \triangle_{h} P$ is given by

$$
\left(\eta^{i}, \nabla_{h} \cdot \mathbf{U}+t^{2} \triangle_{h} P\right)=\left(S^{T} \mathbf{U}_{c}-t^{2} K_{p} P_{c}\right)_{i}
$$

where $S$ is as defined before, $K_{p}=\left(\left(\nabla \eta^{i}, \nabla \eta^{j}\right)\right)$. Similarly, the dual vector of $\nabla_{h} \cdot \mathbf{V}+t^{2} \triangle_{h} Q$ is given by

$$
\left(\eta^{i}, \nabla_{h} \cdot \mathbf{V}+t^{2} \triangle_{h} Q\right)=\left(S^{T} \mathbf{V}_{c}-t^{2} K_{p} Q_{c}\right)_{i} .
$$

If we want to compute the $L^{2}$ inner product

$$
\begin{aligned}
& \left(\nabla_{h} \cdot \mathbf{U}+t^{2} \triangle_{h} P, \nabla_{h} \cdot \mathbf{V}+t^{2} \triangle_{h} Q\right) \\
& \quad=\left(S^{T} \mathbf{U}_{c}-t^{2} K_{p} P_{c}\right)^{T} M^{-1}\left(S^{T} \mathbf{V}_{c}-t^{2} K_{p} Q_{c}\right),
\end{aligned}
$$

at some point we have to use $M^{-1}$, which is not a pleasant thing to do. But $M^{-1}$ is spectrally equivalent to $h^{-2} I$, i.e.,

$$
C h^{-2} \chi^{T} \chi \leq \chi^{T} M^{-1} \chi \leq C h^{-2} \chi^{T} \chi
$$

for any $\chi \in \mathbf{R}^{k}$. Therefore, we define the new inner product $(\cdot, \cdot)_{M}$ on $\left(\Pi_{h} \oplus \mathbf{R}\right) \times$ $\left(\Pi_{h} \oplus \mathbf{R}\right)$ by

$$
(R, R)_{M}=h^{-2} R_{d}^{T} R_{d}
$$

It is easy to see that

$$
C(R, R) \leq(R, R)_{M} \leq C(R, R)
$$

Now

$$
\begin{aligned}
& \gamma\left(\nabla_{h} \cdot \mathbf{U}+t^{2} \triangle_{h} P, \nabla_{h} \cdot \mathbf{V}+t^{2} \triangle_{h} Q\right)_{M} \\
& \quad=\gamma h^{-2}\left(S^{T} \mathbf{U}_{c}-t^{2} K_{p} P_{c}\right)^{T}\left(S^{T} \mathbf{V}_{c}-t^{2} K_{p} Q_{c}\right)
\end{aligned}
$$

is easily computable. Because of (6.1), in the proof of the theorems, we can switch between $(\cdot, \cdot)$ and $(\cdot, \cdot)_{M}$ any time we want to. The matrix corresponding to the right hand side of the last equality is

$$
\mathcal{A}_{2}=\gamma h^{-2}\left(\begin{array}{c}
S \\
-t^{2} K_{p}
\end{array}\right)\left(\begin{array}{ll}
S^{T} & -t^{2} K_{p}
\end{array}\right)
$$

For the other term in the least squares bilinear form (3.8), $h^{2}(A \mathbf{U}-\nabla P, A \mathbf{V}-\nabla Q)$, if linear elements are used, the matrix is

$$
\mathcal{A}_{3}=h^{2}\left(\begin{array}{cc}
0 & 0 \\
0 & K_{p}
\end{array}\right) \text {. }
$$


Therefore the matrix corresponding to the form (3.8) is

$$
\mathcal{A}=\mathcal{A}_{1}+\mathcal{A}_{2}+\mathcal{A}_{3} .
$$

3. Next we consider the preconditioning of the least squares bilinear form (3.8). Recall the stability theorem: For any $(\mathbf{U}, P) \in \mathbf{V}_{h} \times \Pi_{h}$,

$$
\|\mathbf{U}\|_{1}^{2}+\|P\|^{2}+t^{2}\|P\|_{1}^{2}+t^{4}\left\|\triangle_{h} P\right\|^{2} \asymp\langle\langle(P, \mathbf{U}),(P, \mathbf{U})\rangle\rangle,
$$

where $\asymp$ means that the two sides are spectrally equivalent. This tells us that the system can be preconditioned diagonally. We already have a preconditioner of the form

$$
D(\mathbf{U}, \mathbf{U})=\|\mathbf{U}\|_{1}^{2},
$$

namely $B_{h}$, which is used in the least squares formulation. For the other component, $P$,

$$
\begin{aligned}
C(\| P & \left.\left\|^{2}+t^{2}\right\| P\left\|_{1}^{2}+t^{4}\right\| \triangle_{h} P \|^{2}\right) \\
& \leq\left(P-t^{2} \triangle_{h} P, P-t^{2} \triangle_{h} P\right)=\left(\left(I-t^{2} \triangle_{h}\right)^{2} P, P\right) \\
& \leq C\left(\|P\|^{2}+t^{2}\|P\|_{1}^{2}+t^{4}\left\|\triangle_{h} P\right\|^{2}\right) .
\end{aligned}
$$

Hence, what we need is a preconditioner of the operator $\left(I-t^{2} \triangle_{h}\right)^{2}$ on $\Pi_{h}$. Of course, even if $B$ is a preconditioner of $I-t^{2} \triangle_{h}, B^{-2}$ does not have to be spectrally equivalent to $\left(I-t^{2} \triangle_{h}\right)^{2}$. Fortunately, it is proved that such a kind of $B$ does exist [8]. A multigrid V-cycle with variable numbers of smoothing is one of the good choices. Besides, when $t$ is small, $\left(I-t^{2} \triangle_{h}\right)^{2}$ is not really ill conditioned. The numerical experiments suggest that even a $\mathrm{V}$-cycle with constant 2 smoothing will do a very good job. To summarize, we have

$$
\|\mathbf{U}\|_{1}^{2}+\|P\|^{2}+t^{2}\|P\|_{1}^{2}+t^{4}\left\|\triangle_{h} P\right\|^{2} \asymp\left(B_{h}^{-1} \mathbf{U}, \mathbf{U}\right)+\left(B_{P}^{-2} P, P\right),
$$

where $B_{P}$ is a preconditioner of $I-t^{2} \triangle_{h}$, such that $B_{P}^{2}$ is a preconditioner of $\left(I-t^{2} \triangle_{h}\right)^{2}$. If $B_{2}$ is the matrix corresponding to $B_{P}$, then the matrix of the diagonal preconditioner is

$$
\mathcal{B}=\left(\begin{array}{cc}
B_{1} & 0 \\
0 & B_{2}^{2}
\end{array}\right)
$$

\section{NUMERICAL EXPERIMENTS}

Numerical experiments are done for different plate thicknesses on the unit square $\Omega=[0,1] \times[0,1]$. First, the domain is divided into $2^{J} \times 2^{J}$ squares of size $h \times h$, with $h=2^{-J}$. Then, each small square is divided into two triangles by the NWSE diagonal. Continuous piecewise linear elements are used for all the unknowns. As is expected, the numerical solution does converge to the exact solution at the optimal rate without locking. The standard multigrid method is used to compute the solutions of the two elliptic problems. The conjugate gradient method with diagonal preconditioning is used to solve (5.13). The hard work is the tuning of the preconditioner.

In general, the condition number of a block diagonally preconditioned multivariable problem depends heavily on the weighting of the diagonal blocks. Even though the condition number is proved to be independent of the mesh size $h$, the constants can be large and the iteration can behave very poorly if the subsystems are 
unbalanced or the parameters of the preconditioner do not match the parameters of the original problem. For the Reissner-Mindlin plate model (2.1)

$$
a(\boldsymbol{\phi}, \boldsymbol{\psi})+\lambda t^{-2}(\boldsymbol{\phi}-\nabla \omega, \boldsymbol{\psi}-\nabla \mu)=(g, \mu), \quad \forall(\mu, \boldsymbol{\psi}) \in H_{0}^{1}(\Omega) \times \mathbf{H}_{0}^{1}(\Omega),
$$

we define $\widetilde{t}$ and $\widetilde{g}$ by

$$
\widetilde{t}^{-2}=\frac{12\left(1-\nu^{2}\right) \lambda t^{-2}}{E}, \quad \widetilde{g}=\frac{12\left(1-\nu^{2}\right) g}{E},
$$

and then consider the equivalent dimensionless problem

$$
\bar{a}(\boldsymbol{\phi}, \boldsymbol{\psi})+\widetilde{t}^{-2}(\boldsymbol{\phi}-\nabla \omega, \boldsymbol{\psi}-\nabla \mu)=(\widetilde{g}, \mu), \quad \forall(\mu, \boldsymbol{\psi}) \in H_{0}^{1}(\Omega) \times \mathbf{H}_{0}^{1}(\Omega) .
$$

The meaning of $\bar{a}(\cdot, \cdot)$ is clear in the context. Instead of four parameters $E, \lambda, \nu$, and $t$, we now need to deal with only two parameters $\nu$ and $\widetilde{t}$. The parameter $\gamma$ in the form (3.8) needs to be tuned to balance the subsystems corresponding to the two equations of the Stokes-like problem. For the rest of this section, we will use $a(\cdot, \cdot)$ for $\bar{a}(\cdot, \cdot), t$ for $\widetilde{t}$, and $g$ for $\widetilde{g}$, just to simplify the notation.

In the diagonal preconditioner $\left(\begin{array}{cc}B_{h} & 0 \\ 0 & B_{P}^{2}\end{array}\right), B_{h}: \mathbf{H}_{0}^{1}(\Omega) \longrightarrow \mathbf{V}_{h}$ is a multigrid $\mathrm{V}$-cycle preconditioner for the operator $-\triangle$ on $\mathbf{H}_{0}^{1}(\Omega)$. The number of smoothings can be one, but we chose two because we found that the case of two smoothings is more efficient in terms of total work. $B_{P}$ is a multigrid V-cycle preconditioner for $I-t^{2} \triangle_{h}$ on $\Pi_{h}$. We also choose two smoothings in practice, although more smoothing is needed on the coarse grids to prove that $B_{P}^{-2}$ is spectrally equivalent to $\left(I-t^{2} \triangle_{h}\right)^{2}$. The numerical experiment shows that three smoothings work better when $h<<t$.

Let $X$ be the solution of $\mathcal{A} X=F, X_{m}$ be the iterates, $R^{m}=F-\mathcal{A} X_{m}$ be the residual. Then there are $0<C_{0}<C_{1}$, independent of $h$, such that

$$
C_{0}\left(\mathcal{A}\left(X-X_{m}\right), X-X_{m}\right) \leq\left(\mathcal{B} R^{m}, R^{m}\right) \leq C_{1}\left(\mathcal{A}\left(X-X_{m}\right), X-X_{m}\right),
$$

and so

$$
\frac{C_{0}}{C_{1}} \frac{\left(\mathcal{B} R^{m}, R^{m}\right)}{\left(\mathcal{B} R^{0}, R^{0}\right)} \leq \frac{\left(\mathcal{A}\left(X-X_{m}\right), X-X_{m}\right)}{\left(\mathcal{A}\left(X-X_{0}\right), X-X_{0}\right)} \leq \frac{C_{1}}{C_{0}} \frac{\left(\mathcal{B} R^{m}, R^{m}\right)}{\left(\mathcal{B} R^{0}, R^{0}\right)}
$$

Therefore

$$
\frac{\left(\mathcal{B} R^{m}, R^{m}\right)}{\left(\mathcal{B} R^{0}, R^{0}\right)} \leq \varepsilon
$$

is used to stop the iteration. In the conjugate gradient method, $\left(\mathcal{B} R^{m}, R^{m}\right)$ is computed as part of the iteration, so the error estimator is free of cost.

To reveal the real error reduction rate and condition number of the preconditioned system, we choose $\varepsilon=10^{-8}$. It is well known that the error reduction in the first few iterations does not reflect the condition number of the system. Too large a value of $\varepsilon$ might produce an unreliable estimate of the condition number.

Table 1 reports the iteration numbers of the conjugate gradient iterations solving (5.13) for mesh size $h=1 / 8,1 / 16,1 / 32,1 / 64,1 / 128$ and plate thickness $t=$ $0,0.001,0.01,0.1,1.0$, with $\nu=0.25, \gamma=0.36, \varepsilon=10^{-8}$. The condition number of $\mathcal{B A}$ can be estimated by

$$
K(\mathcal{B A}) \leq((1+\theta) /(1-\theta))^{2}, \quad \theta=\varepsilon^{\frac{1}{m}}
$$

where $m$ is the iteration number. For all the cases in the table, $K(\mathcal{B A}) \in(7.4,26.8)$. The condition numbers are uniformly bounded, although they are larger when 
TABLE 1. Iteration numbers of the PCG, $\nu=0.25, \gamma=0.36, \varepsilon=10^{-8}$

\begin{tabular}{|r|r|r|r|r|r|}
\hline$h=$ & $2^{-3}$ & $2^{-4}$ & $2^{-5}$ & $2^{-6}$ & $2^{-7}$ \\
\hline$t=0.000$ & 24 & 26 & 30 & 34 & 35 \\
\hline$t=0.001$ & 24 & 26 & 30 & 34 & 35 \\
\hline$t=0.010$ & 25 & 27 & 28 & 34 & 39 \\
\hline$t=0.100$ & 29 & 42 & 41 & 40 & 41 \\
\hline$t=1.000$ & 33 & 40 & 43 & 45 & 47 \\
\hline
\end{tabular}

$h<<t$. The reason for this is reflected in the proof of the stability theorem, which we use to construct the diagonal preconditioner; the constants change when $t \neq 0$.

\section{REFERENCES}

1. D. Arnold and R. Falk, A uniformly accurate finite element method for the Reissner-Mindlin plate, SIAM J. Numer. Anal. 26 (1989), pp. 1276-1290. MR 91c:65068

2. D. Arnold and R. Falk, Analysis of a linear-linear finite element for the Reissner-Mindlin plate model, Math. Models and Methods in Applied Sciences, 7 (1997), pp. 217-238. MR 98b:73034

3. D. Arnold, R. Falk and R. Winther, Preconditioning in H(div) and applications, Math. Comp. 66 (1997), pp. 957-984. MR 97i:65177

4. D. Arnold, R. Falk and R. Winther, Preconditioning discrete approximations of the ReissnerMindlin plate model, RAIRO Modél. Math. Anal. Numér. 31 (1997), pp. 517-557. CMP 97:15

5. J. Bramble, Multigrid Methods, Pitman Research Notes in Mathematics Series, Longman Scientific \& Technical, London. Copublished with John Wiley \& Sons, Inc., New York, 1993. MR 95b:65002

6. J. Bramble, R. Lazarov and J. Pasciak, A least-square approach based on a discrete minus one inner product for first order systems, Math. Comp., 66 (1997), pp. 935-955. MR 97m:65202

7. J. Bramble and J. Pasciak, Least-squares methods for Stokes equations based on a discrete minus one inner product, J. Comp. Appl. Math., 74 (1996), pp. 155-173. CMP 97:07

8. J. Bramble and J. Pasciak, New convergence estimates for multigrid algorithms, Math. Comp. 49(1987), pp. 311-329. MR 89b:65234

9. S. Brenner, Multigrid methods for parameter dependent problems, RAIRO Modél. Math. Anal. Numér. 30 (1996), pp. 265-297. MR 97c:73076

10. S. Brenner and L. Scott, The Mathematical Theory of Finite Element Methods, SpringerVerlag, 1994. MR 95f:65001

11. F. Brezzi and M. Fortin, Numerical approximation of Mindlin-Reissner plate, Math. Comp. 47 (1986), pp. 151-158. MR 87g:73057

12. F. Brezzi, M. Fortin and R. Stenberg, Error analysis of mixed-interpolated elements for Reissner-Mindlin plates, Math. Models and Methods in Applied Sciences, 1 (1991), pp. 125151. MR 92e: 73030

13. R. Duran and E. Liberman, On mixed finite element methods for Reissner-Mindlin plate models, Math. Comp., 58 (1992), pp. 561-573. MR 92f:65135

14. T. Hughes and L. Franca, A stable bilinear element formulation for Reissner-Mindlin plate theory: Uniform convergence of all higher-order spaces, Comp. Meths. Appl. Mech. Engrg., 67 (1988), pp. 223-240. MR 89g:73033

15. E. Onate, F. Zarate and F. Flores, A simple triangular element for thick and thin plate and shell analysis, Int. J. Numer. Method. Engrg., 37 (1994), pp. 2569-2582.

16. P. Peisker, A multigrid method for Reissner-Mindlin plates, Numer. Math., 59 (1991), 511528. MR 92g:73083

17. V. Girault and P. Raviart, Finite element approximation of the Navier-Stokes equations. Lecture Notes in Math. \#749, Springer-Verlag, New York, 1981. MR 83b:65122 
18. R. Stenberg, A new finite element formulation for the plate bending problem, Proceedings of the International Conference on Asymptotic Methods for Elastic Structures, Lisbon, 1993. MR 96k:73084

Department of Mathematics, Texas A\&M University, College Station, Texas 77840

E-mail address: bramble@math.tamu.edu

Institute for Scientific Computation, Texas A\&M University, College Station, Texas 77840

E-mail address: tsun@math.tamu.edu 